\title{
CUERPO EXTRAÑO EN LA VIA AÉREA EN PEDIATRÍA
}

\author{
Foreign-body aspiration in children
}

\author{
Dr. Julio Maggiolo M'1, Dra. Lilian Rubilar 01, Dr. Guido Girardi B² \\ 1. Pediatra Especialista en Enfermedades Respiratorias. Unidad Broncopulmonar. Hospital Exequiel González Cortés. \\ 2. Pediatra Especialista en Enfermedades Respiratorias Profesor Titular de Pediatría Universidad de Chile. Unidad Broncopulmonar. \\ Hospital Exequiel González Cortés.
}

\begin{abstract}
FOREIGN-BODY ASPIRATION IN CHILDREN
It is a frequent pediatric event and a common cause of morbidity and mortality in children, requiring accurate diagnosis and proper treatment. Diagnosis is usually based on clinical manifestations such as cough, dyspnea. stridor, respiratory distress and asymmetric decreased air entry or unilateral wheeze. Choking crisis may be absent or minimized. Chest X-ray may have no abnormalities or show signs such as asymmetrical hyperinflation, massive, fixed or changing atelectasis. Occasionally, hyperinflation or obstructive emphysema with atelectasis can be present in the same hemithorax. Inspiratory and expiratory chest X-rays show ipsilateral mediastinum movements. Most foreign bodies are not visible to chest $\mathrm{X}$-ray exploration.

Acute suffocation must be treated with Heimlich procedure. In later stages, diagnosis and treatment require rigid bronchoscopy. Prognosis is fairly positive with an early and complete foreign-body extraction
\end{abstract}

Key words: foreign-body, aspiration, Heimlich maneuver, airway obstruction

\section{RESUMEN}

La aspiración de un cuerpo extraño (CE) a la vía aérea en pediatría es un accidente frecuente, presenta importante morbimortalidad, por lo que requiere un diagnóstico y tratamiento oportuno. El diagnóstico usualmente se establece mediante hallazgos clínicos, siendo los más frecuentes tos, sindrome de penetración, y con menor frecuencia dificultad respiratoria, estridor, sibilancias unilaterales. La radiografía de tórax puede ser normal 0 alterada, mostrando hiperinsuflación unilateral, atelectasia u otros signos. La mayoría de los CE son radiotransparentes.

Durante la fase aguda debe practicarse la maniobra de Heimlich, en cambio en la fase crónica el diagnóstico y tratamiento definitivo se hace mediante broncoscopía.

La prevención es fundamental evitando que niños menores manipulen objetos pequeños, advertir el riesgo de asfixia por aspiración en los envases de alimentos riesgosos y de juguetes de pequeño tamaño.

El pronóstico es bueno si la extracción del cuerpo extraño es precoz y completa.

Palabras clave: cuerpo extraño, aspiración, maniobra de Heimlich, obstrucción de las vías aéreas.

\section{INTRODUCCIÓN}

En pediatría la aspiración de un cuerpo extraño (CE) a la vía aérea es un accidente frecuente, especialmente entre los 18 meses y 3 años de edad, pudiendo presentarse como una emergencia, causando importante morbilidad y en ocasiones mortalidad, requiriendo por lo tanto diagnóstico y tratamiento precoz (1).

En un alto porcentaje de casos, el niño se encuentra sólo en el momento de aspirar el cuerpo extraño, por esta razón se debe tener siempre presente este diagnóstico en un niño con dificultad respiratoria. Importante es la educación impartida a los padres y personas que cuidan niños, al personal de salud en lo que respecta a evitar este accidente, cuando se debe sospechar, además del manejo precoz y adecuado $(2,3)$.

\section{Correspondencia:}

Dr. Julio Maggiolo Massone

Hospital Exequiel González Cortés

Ramón Barros Luco 3301, Santiago.

E-mail : maggiolojulio@gmail.com

\section{ORIGEN DEL CUERPO EXTRAÑO}

El objeto aspirado más frecuente es de origen vegetal, como maní, almendras, porotos, trozos de fruta, maíz, arroz. También han sido descritos trozos de carne, botones, tapa de lápices, agujas, aros, tornillos, juguetes, alfileres, cáscara de huevo, trozos de globo, bolitas. Estos dos últimos objetos se han asociado más frecuentemente a casos mortales, debido a obstrucción completa de la vía aérea. Los objetos inorgánicos son más frecuentes en el grupo de mayor edad, como la tapa de lápiz a pasta (4). Los objetos pequeños como las piezas de juguetes han disminuido su incidencia gracias a las campañas de prevención $(2,3)$. Por otra parte los tapones de mucus endobronquiales y en casos más extremos la bronquitis plástica, que consiste en la obstrucción bronquial por un tapón que adopta la forma del árbol traqueobronquial debe diferenciarse de un CE.

\section{EPIDEMIOLOGÍA Y FACTORES DE RIESGO}

De todos los accidentes mortales en niños menores de 4 años de edad el 7\% son debidos a aspiración de CE. Esta cifra alcanza el $40 \%$ en menores de 1 año. En cuanto a la 
morbilidad se debe tomar en cuenta la encefalopatía hipóxica, si el tratamiento no se hace en forma oportuna y adecuada (5). Los niños presentan características que favorecen la aspiración de un CE: a partir de los 6 meses llevan objetos a la boca, entre los 8 y 10 meses son capaces de hacer pinza entre el índice y el pulgar, curiosidad, inmadurez de la masticación-deglución y luego del año de edad la capacidad de deambulación. Por todos estos factores la mayor incidencia es en menores de 3 años (80\% de los casos), especialmente entre 1 y 2 años, con un segundo peak alrededor de los 11 años de edad, más frecuente en varones (6). Por otro lado comer cuando se está haciendo alguna actividad física como correr, aumenta las probabilidades de aspiración (7).

Las personas mayores como hermanos y padres debido a ignorancia o por diversión introducen comida u otros objetos en la boca del niño, potencialmente aspirables.

En el niño mayor y adolescentes los factores que se asocian a aspiración son parecidos a los adultos, como la presencia de patología neurológica, pérdida de conciencia y abuso de alcohol o drogas $(6,7)$.

\section{PATOGENIA}

Al contactar el CE con la mucosa de la laringe 0 tráquea se produce el llamado síndrome de penetración, que se caracteriza por una crisis de asfixia por espasmo de la glotis. Se desencadena tos expulsiva que puede eliminar el CE. Si este sobrepasa la glotis, se localizará en sitios inferiores de la vía aérea, dando sintomatología menos evidente (8). La mayoría de los CE son pequeños impactándose en los bronquios. El paciente puede permanecer asintomático por días a meses, incluso años, según la localización, el grado de obstrucción e inflamación (8).

En relación a las vías aéreas, el CE impactado puede dar lugar a la formación de granulomas, mucho menos frecuente son la perforación traqueal o bronquial. También se pueden originar bronquiectasias reversibles si el tratamiento es oportuno, en cambio pueden ser irreversibles si el objeto no es extraido.

La obstrucción bronquial parcial produce hiperinsuflación localizada, debido a un mecanismo de válvula, permitiendo la entrada de aire y dificultando su salida. En otras ocasiones el CE migra de un lóbulo a otro, produciento atelectasia cambiante. La atelectasia masiva ocurre si la obstrucción bronquial es completa, especialmente la producida por CE de origen vegetal, debido a que aumentan de tamaño al absorber agua. La atelectasia aireada se produce por entrada y salida parcial de aire, en tanto la hiperinsuflación y atelectasia en el mismo campo pulmonar se debe a un mecanismo de válvula y obstrucción bronquial respectivamente (9). El CE induce hipersecreción bronquial y posteriormente la sobreinfección puede producir neumonía (8).

\section{MANIFESTACIONES CLÍNICAS}

El signo más frecuente es la tos persistente (90-95\%), siendo poco específica, el síndrome de penetración está presente hasta en un $80 \%$ de los casos, con una sensibilidad de hasta
$97 \%$ y especificidad de $63 \%$. Este dato tan característico no es referido por los padres en todos los casos, se debe preguntar en forma dirigida. Se ha descrito que hasta en un $88 \%$ de casos con diagnóstico tardío los padres habían presenciado el episodio (9). Menos frecuentes son fiebre (30\%), dificultad respiratoria $(15 \%)$, estridor $(15 \%)$, disfonía $(15 \%)$, hemoptisis y cianosis $(5-8)$.

En la auscultación el hallazgo más frecuente es la disminución localizada del murmullo pulmonar (65\%), sibilancias $(45 \%)$ y crepitaciones unilaterales $(25 \%)(7,8)$.

Aunque la exploración física tiene una alta especificidad, hasta un $10 \%$ de los casos es normal, por lo que se debe hacer una anamnesis detallada. Se dice que la presencia de la tríada de tos, sibilancias localizadas e hipoventilación está presente en un $50 \%$ aproximadamente $(7,8)$.

Según la localización del CE se describen manifestaciones clínicas características (5-8):

Laringe: el CE que causa obstrucción produce dificultad respiratoria, estridor inspiratorio y tiraje, en cambio si es móvil laringitis persistente o recurrente.

Tráquea: un CE obstructivo induce estridor inspiratorio y espiratorio, dificultad respiratoria y retracción de partes blandas y si es móvil traqueitis persistente o recurrente.

- Bronquios: luego del síndrome de penetración, el paciente puede permanecer asintomático por un período de tiempo variable. Los CE que permanecen largo tiempo ocasionan neumonías 0 atelectasias de evolución tórpida, persistentes 0 recurrentes en la misma ubicación e incluso bronquiectasias (8). En la Tabla 1 se describen las ubicaciones del CE encontradas en una serie nacional, en la cual la mayoría era bronquial (50\% del total en bronquio derecho)(9).

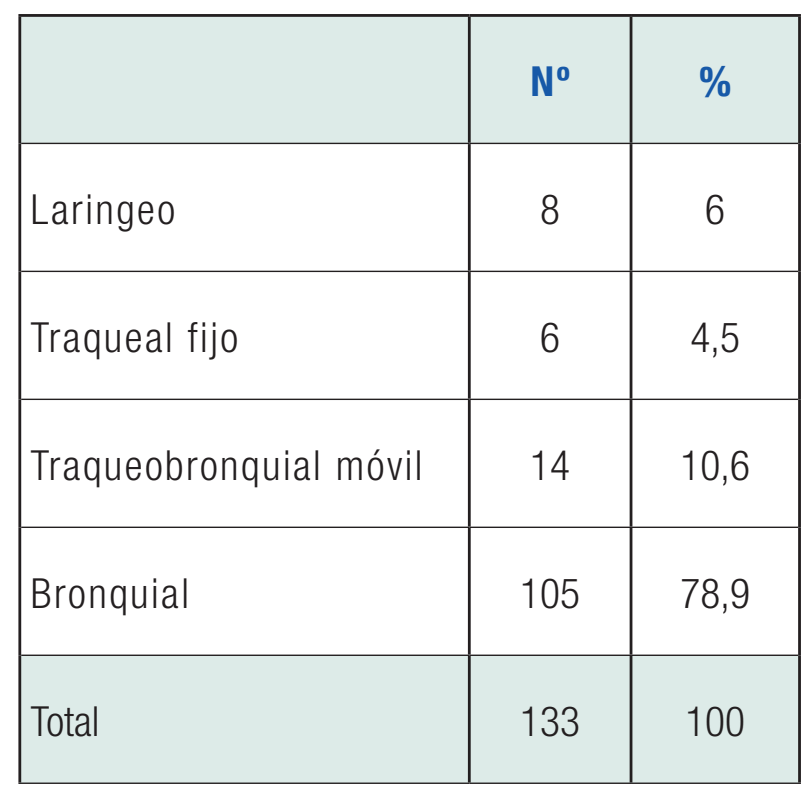

Tabla 1.- Localización según broncoscopía (9) 


\section{DIAGNÓSTICO}

Este es sencillo en aquellos casos en los que los padres o cuidadores han observado el episodio de síndrome de penetración y se dificulta cuando el niño ya se encuentra en la fase asintomática posterior. Es en este segundo caso en que la anamnesis, el examen físico minucioso y las pruebas complementarias deben conducir al diagnóstico y extracción precoz del CE con el objeto de evitar secuelas (8).

La historia tiene una sensibilidad alta (80-90\%), pero es poco específica, algo similar ocurre con el examen físico, se deben practicar otras exploraciones diagnósticas, como radiografía (Rx) tórax, la que puede ser normal entre 12$25 \%$ de los casos. Si la sospecha es alta se debe realizar una fibrobroncoscopía (FBC) con el fin de confirmar o descartar el diagnóstico, procedimiento considerado gold standard $(10,11)$.

\section{Radiografía de tórax y Radioscopía}

Desde el punto de vista radiológico la mayoría de los CE son radiolúcidos, cuando son radioopacos se puede observar el objeto aspirado $(3-23 \%)(9,10)$.

La hiperinsuflación unilateral es producida por mecanismo de válvula, haciéndose más visible al tomar la radiografía en espiración (Figura1) (10). La radioscopía se debe hacer en inspiración y espiración, lo que evidencia el bamboleo mediastínico, durante la inspiración el mediastino se desplaza hacia el lado donde se encuentra el CE, en cambio durante la espiración el mediastino se desplaza hacia el lado opuesto (Figura 2A y 2B) $(9,10)$.

Según la ubicación del CE puede existir atelectasia masiva por obstrucción bronquial completa. Se describen dos signos prácticamente patognómonicos de aspiración de CE: la atelectasia e hiperinsuflación en el mismo campo pulmonar (Figura 3) y la atelectasia aireada (Figura 4) (9). Cuando el CE migra en el árbol bronquial se puede observar hiperinsuflación y atelectasias cambiantes, posteriormente puede manifestarse como atelectasia o neumonía persistente 0 recurrente en la misma ubicación (10).

Los hallazgos radiológicos más frecuentemente encontrados se muestran en la Tabla 2 (9).

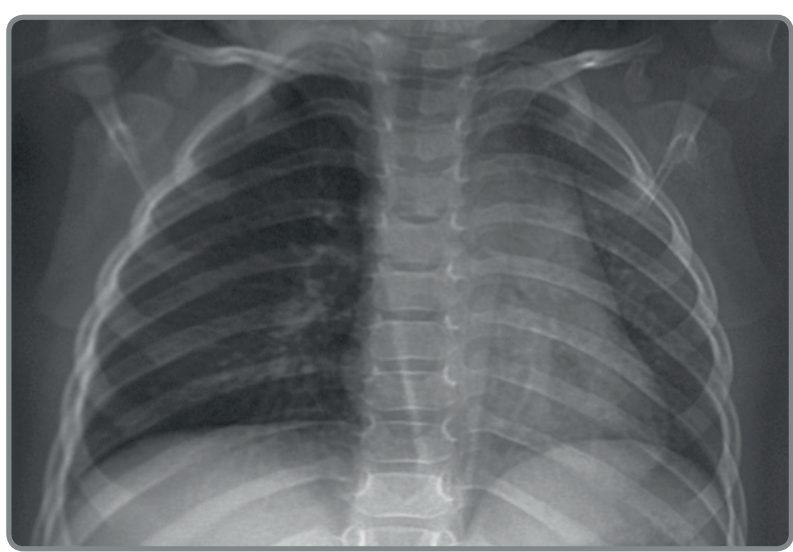

Figura 1. Rx tórax: insuflacíón pulmonar derecha.

Paciente de 1año3meses de edad. Poroto en bronquio fuente derecho

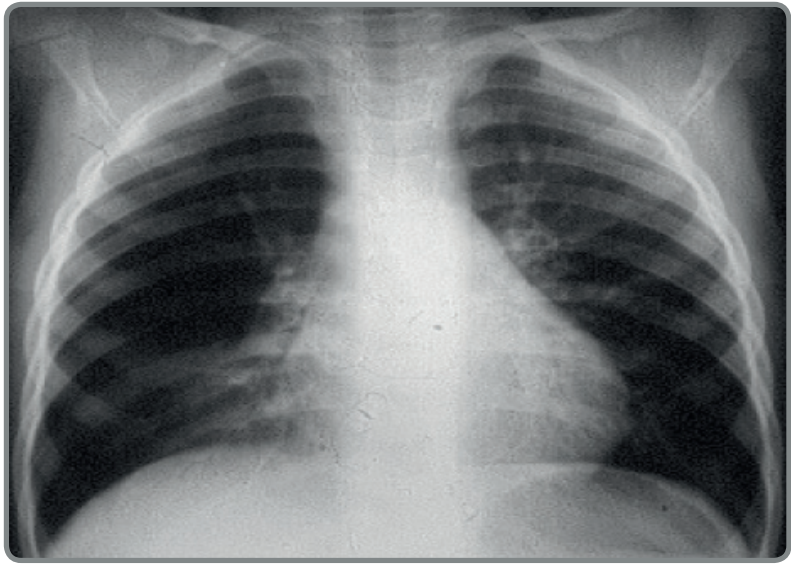

Figura 2A. Rx tórax (inspiración): desviación del mediastino hacia el hemitórax derecho, insuflación pulmonar ipsilateral más atelectasia aireada lóbulo medio.

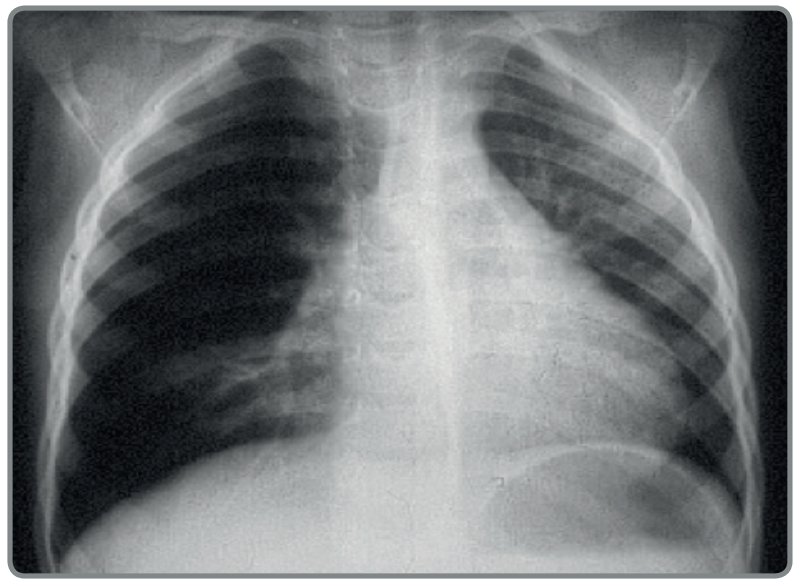

Figura 2B. Rx tórax (espiración): desviación del mediastino hacia el hemitórax izquierdo, insuflación pulmonar derecha más atelectasia aireada lóbulo medio.

Paciente de 4 años de edad. Uva en bronquio del lóbulo medio.

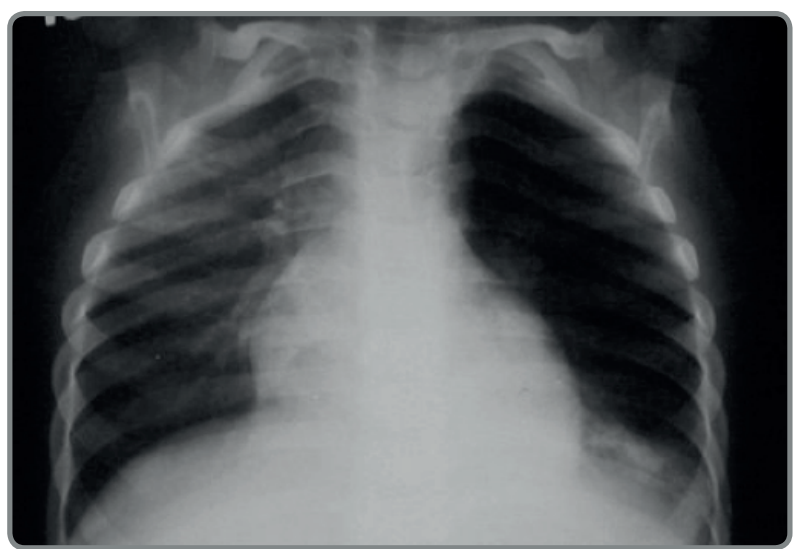

Figura 3. Rx tórax: insuflación pulmonar izquierda, más atelectasia aireada del lóbulo inferior izquierdo.

Paciente de 3 años de edad. Tapa de lápiz BIC en bronquio fuente izquierdo. 


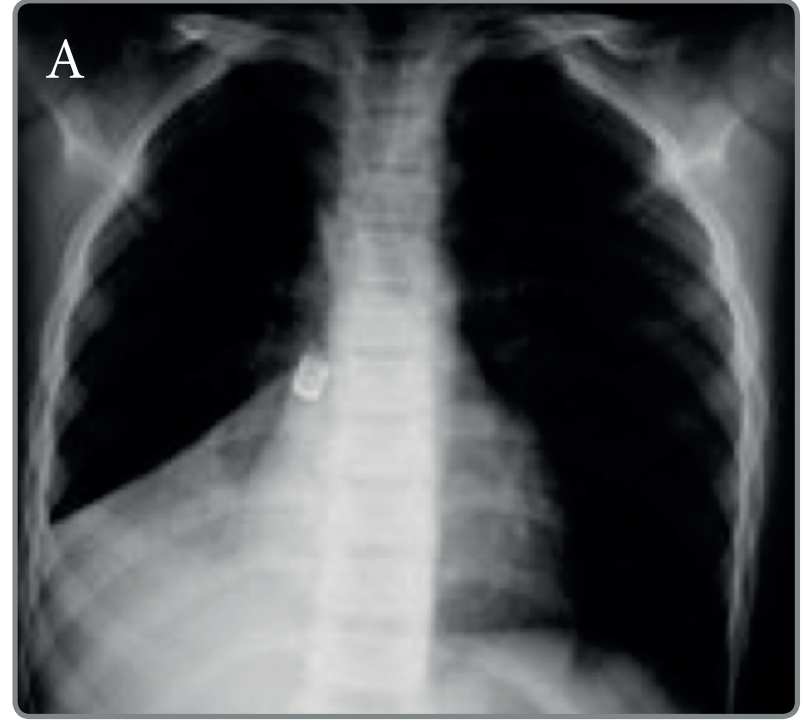

Figura 4A. Rx tórax: objeto radiopaco a derecha, atelectasia aireada lóbulo inferior derecho.

Figura 4B. Cuerpo extraño metálico. Paciente de 3 años de edad. Ampolleta en lóbulo inferior derecho.

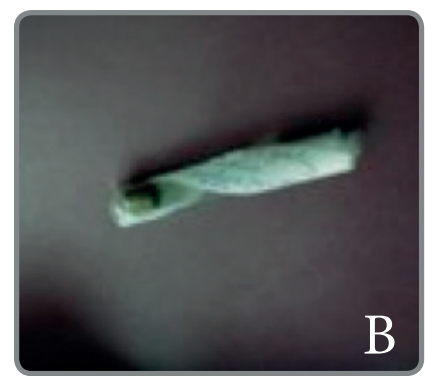

\begin{tabular}{|l|c|c|}
\hline & $\mathbf{N}^{\mathbf{0}}$ & $\%$ \\
\hline Objeto radiopaco & 31 & 23,3 \\
\hline $\begin{array}{l}\text { Hiperinsuflacion } \\
\text { pulmonar unilateral }\end{array}$ & 29 & 21,8 \\
\hline $\begin{array}{l}\text { Hiperinsuflacion + } \\
\text { atelectasia unilateral }\end{array}$ & 24 & 18 \\
\hline Atelectasia lobar & 17 & 12,8 \\
\hline Atelectasia pulmonar & 9 & 6,8 \\
\hline Atelectasia aireada & 8 & 6 \\
\hline Neumonía & 1 & 3 \\
\hline Normal & 15 & 11,3 \\
\hline
\end{tabular}

Tabla 2. Hallazgos radiológicos (9).

(Algunos pacientes presentaban más de 1 hallazgo radiológico)
La broncoscopía rígida (BR) confirma el diagnóstico y es terapéutica de los CE en la vía aérea, en el caso que no se encuentre 0 existen dudas de su extracción completa, se debe practicar broncoscopía flexible, con el objeto de visualizar bronquios segmentarios y los lóbulos superiores (11-13).

\section{TRATAMIENTO}

Cuando el CE se ubica en bronquios se debe proceder a su extracción mediante broncoscopía rígida, realizada por médicos con experiencia, por la dificultad técnica y el riesgo que implica, debido a que el CE puede soltarse accidentalmente y penetrar en otro bronquio o producirse obstrucción aguda de la tráquea o laringe (12-16).

El diagnóstico y la extracción debe hacerse en un solo tiempo, en pabellón, contando con ambos tipos de broncoscopio. El flexible se utiliza eventualmente para identificar y localizar el $\mathrm{CE}$, y posterior a la extracción para revisar la vía aérea. (11-17).

Si el CE se encuentra en la vía aérea superior se produce asfixia aguda, una emergencia médica, debiendo ser tratada oportunamente mediante maniobra de Heimlich. Esta debe enseñarse a estudiantes de medicina, carreras paramédicas y en cursos de primeros auxilios a personas que cuidan niños, como padres, profesores, educadoras de párvulos (8).

\section{PRONÓSTICO}

En general es bueno, incluso si el diagnóstico es tardío, cuando el CE es extraído completamente. La regresión de las atelectasias e incluso de algunas bronquiectasias cilíndricas es completa, en cambio si estas son saculares es más difícil que se resuelvan.

La permanencia de un CE en bronquio por tiempo prolongado se relaciona con la presencia de sibilancias unilaterales, confundidas con síndrome bronquial obstructivo 0 asma bronquial, neumonías recurrentes y atelectasias persistentes y localizadas, que no ceden al tratamiento convencional y bronquiectasias de etiología desconocida (8).

En la serie de Girardi y cols el tiempo de permanencia del CE en la vía aérea antes de su extracción endoscópica fue de 1 día a 10 años (9).

\section{PREVENCIÓN}

La educación resulta fundamental en lo que se refiere a evitar que niños menores manipulen objetos pequeños, enseñar la realización de la maniobra de Heimlich a la población general, padres, cuidadores de niños, estudiantes de enfermería, medicina. Advertir en los envases de alimentos riesgosos (maní, porotos, caramelos, etc) y de juguetes de pequeño tamaño del riesgo de asfixia por aspiración $(2,3)$.

Los autores declaran no presentar conflicto de interés. 


\section{REFERENCIAS}

1. Ayed AK, Jafar AM, Owayed A. Foreign body aspiration in children: diagnosis and treatment. Pediatr Surg Int 2003; 19: 485-8

2. Karatzanis A, Vardouiotis J, Moschandreas E, Prokopakis E, Papadakis D, Kyrmizakis $J$ et al. The risk of foreign body aspiration in children can be reduced with proper education of the general population. Int J Pediatr Otorhinolaryngol 2007; 71: $311-5$

3. WISQARS Leading causes of death reports, 1999-2001. National Center for Injury Prevention and Control. Disponible en: webapp.cdc. gov/sasweb/ncipc/lead-caus 10.html.

4. Zhijun CJ, Fugao Z, Niankai Z, Jingjing C. Therapeutic experience from 1428 patients with pediatr tracheobronchial foreign body. J Pediatr Surg 2008; 43: 718

5. Martínez Nadal J, Figuerola Mulet J, Tomás Barberan M. Cuerpos extraños laringotraqueobronquiales en la infancia. En: Tomás M, Bernal M. Tratado de Otorrinolangología Pediátrica. Girona: Ed. Gráficas Alzamora; 2000, p 433-436

6. Even L, Heno N, Talmon Y, Samet E, Zonis Z, Kugelman A. Diagnostic evaluation of foreign body aspiration in children: a prospective study. J Pediatr Surg 2005; 40: 1122

7. Sánchez Echániz, Pérez J, Mintegui S, Benito J, López-Alvarez P. Aspiración de cuerpo extraño en la infancia. An Esp Pediatr 1996; 45: 365-8

8. Sánchez Solís de Queral M. Aspiración aguda y aspiración crónica. En: Cobos N, Pérez Yarza EG. Tratado de Neumología infantil. $2^{a}$ ed. Madrid: Ergon; 2009, p589-607

9. Girardi G, Contador Ana M, Castro-Rodríguez JA. Two New Radiological Findings to Improve the Diagnosis of Bronchial Foreign-Body Aspiration in Children. Pediatr Pulmonol 2004; 38 : 261-4

10. Assefa D; Amin, Stringel G; Dozor AJ. Use radiographs in the diagnosis of foreign body aspiration in young children: Pediatr Emerg Care 2007; 23: 154

11. Dikensoy 0, Usalan C, Filiz A. Foreign body aspiration: clinical utility of flexible broncoschoscopy. Postgrad Med J 2002; 78: 399-403

12. Lan-fang Tang, Ying-Chun Xu, Ying-Shuo Wang, Cai-Fu Wang, Guo-Hong Zhu et al. Airway foreign removal by rigid bronchoscopy: experience with 1027 children during 20002008. World J Pediatr 2009; 5: 191-195

13. Ramírez-Figueroa JL, Gochicoa-Rangel LG, RamírezSan Juan DH, Vargas MH. Foreign body removal by fiberoptic bronchoscopy in infants and children. Pediatr Pulmonol 2005; 40: 392-397

14. Swanson KL. Airway forign bodies: what's new? Semin Respir Crit Care Med. Int Pulmonol 2004; 25: 405-11

15. Swanson KL, Prakash UB, Midthun DE,Edell ES, Utz JP, McDougall JC et al. Flexible bronchoscopic management of airway foreign bodies in children. Chest 2002; 121: 1695-700

16. Shlomo Cohen, Avraham Avital, Simon Godfrey, Menachem Gross, Eitan Kerem, Chaim Springer. Suspected foreign body inhalation in children: what are the indications for bronchoscopy? J Pediatr 2009; 155: 276-80

17. Figueroa J, Peña JA. Cuerpos extraños en la vía aérea. En: Andrés A, Valverde J. Manual de Neumología Pediátrica. 1era edición. Madrid. Ed. Panamericana; 2011, p 405-413 